\title{
BMJ Open Similarities and differences in the associations between patient safety culture dimensions and self-reported outcomes in two different cultural settings: a national cross-sectional study in Palestinian and Belgian hospitals
}

\author{
Shahenaz Najjar, ${ }^{1,2}$ Elfi Baillien, ${ }^{3}$ Kris Vanhaecht, ${ }^{4}$ Motasem Hamdan, ${ }^{5}$ \\ Martin Euwema, ${ }^{6}$ Arthur Vleugels, ${ }^{2}$ Walter Sermeus, ${ }^{2}$ Ward Schrooten, ${ }^{7}$ \\ Johan Hellings, ${ }^{7}$ Annemie Vlayen ${ }^{7}$
}

To cite: Najjar S, Baillien $\mathrm{E}$, Vanhaecht $\mathrm{K}$, et al. Similarities and differences in the associations between patient safety culture dimensions and self-reported outcomes in two different cultural settings: a national cross-sectional study in Palestinian and Belgian hospitals. BMJ Open 2018;8:e21504. doi:10.1136/ bmjopen-2018-021504

- Prepublication history for this paper is available online. To view these files please visit the journal online (http://dx.doi. org/10.1136/bmjopen-2018021504).

Received 4 January 2018 Revised 20 May 2018 Accepted 15 June 2018

Check for updates

(c) Author(s) (or their employer(s)) 2018. Re-use permitted under CC BY-NC. No commercial re-use. See rights and permissions. Published by BMJ.

For numbered affiliations see end of article.

Correspondence to Dr Shahenaz Najjar; shnajjar@gmail.com

\section{ABSTRACT}

Objectives To investigate the relationships between patient safety culture (PSC) dimensions and PSC selfreported outcomes across different cultures and to gain insights in cultural differences regarding PSC.

Design Observational, cross-sectional study.

Setting Ninety Belgian hospitals and 13 Palestinian hospitals.

Participants A total of 2836 healthcare professionals matched for profession, tenure and working hours. Primary and secondary outcome measures The validated versions of the Belgian and Palestinian Hospital Survey on Patient Safety Culture were used. An exploratory factor analysis was conducted. Reliability was tested using Cronbach's alpha $(\alpha)$. In this study, we examined the specific predictive value of the PSC dimensions and its self-reported outcome measures across different cultures and countries. Hierarchical regression and bivariate analyses were performed.

Results Eight PSC dimensions and four PSC selfreported outcomes were distinguished in both countries. Cronbach's $\alpha$ was $\alpha \geq 0.60$. Significant correlations were found between PSC dimensions and its self-reported outcome ( $p$ value range $<0.05$ to $<0.001$ ). Hierarchical regression analyses showed overall perception of safety was highly predicted by hospital management support in Palestine $(\beta=0.16, p<0.001)$ and staffing in Belgium $(\beta=0.24, p<0.001)$. The frequency of events was largely predicted by feedback and communication in both countries (Palestine: $\beta=0.24, p<0.001$; Belgium: $\beta=0.35$, $p<0.001)$. Overall grade for patient safety was predicted by organisational learning in Palestine $(\beta=0.19, p<0.001)$ and staffing in Belgium $(\beta=0.19, p<0.001)$. Number of events reported was predicted by staffing in Palestine $(\beta=-0.20$, $\mathrm{p}<0.001)$ and feedback and communication in Belgium $(\beta=0.11, p<0.01)$

Conclusion To promote patient safety in Palestine and Belgium, staffing and communication regarding errors should be improved in both countries. Initiatives to improve hospital management support and establish constructive
Strengths and limitations of this study

Our study enables us to draw conclusions regarding the varying associations among patient safety culture (PSC) dimensions and self-reported patient safety outcomes across different cultural settings.

- This is the first study to explore the predictive value of the Hospital Survey on Patient Safety Culture (HSOPSC) in matched samples from two different countries.

- Using a matched samples study design is useful, allowing researchers to establish a good degree of validity and to investigate a rationalised and focused research question.

- Our results verify the international and cross-cultural validity of the HSOPSC; however, more studies are needed to provide further evidence in this regard.

- Our study relied exclusively on subjective self-reported measures; future research on linking actual adverse events data with PSC dimensions objectively is required to clarify this relationship.

learning systems would be especially beneficial for patient safety in Palestine. Future research should address the association between safety culture and hard patient safety measures such as patient outcomes.

\section{BACKGROUND}

Patient safety is a major focus in the improvement of the quality of healthcare. ${ }^{1-3}$ This has led to an increased interest in patient safety culture (PSC) assessments in healthcare organisations. ${ }^{4}$ Clinical outcomes and adverse event rates have been used as indicators of patient safety in previous studies. ${ }^{5}$ Earlier research has demonstrated a link between organisational culture and hospital 
outcomes, adverse event rates, ${ }^{6-8}$ financial performance ${ }^{9}$ or patient satisfaction. ${ }^{10}$ The development of a positive PSC may help to integrate the concern for safety into the daily functioning of organisations and into the routines of individuals and teams. ${ }^{6}$ In this study, PSC is defined as the presence of values, beliefs, norms, behaviours and attitudes that may contribute to patient safety. ${ }^{11}$ Hospitals that invest in PSC may enable staff to prevent and solve safety problems and to learn collectively from problems that occur at the frontline of healthcare delivery. ${ }^{8} 1213$ Several studies have highlighted the perception of patient safety and staff attitudes towards safety. ${ }^{14-16}$ Others have reported on the psychometric evaluation of PSC tools. ${ }^{17-21}$

A range of tools has been developed to measure PSC, for example, PSC in healthcare organisations, hospital surveys on patient safety and safety attitude questionnaires. ${ }^{22}{ }^{23}$ These instruments can be used elicit patient safety-related assessments by healthcare staff at: (1) a hospital level, (2) a unit level and (3) a professional level. ${ }^{16} 24$ The original American Hospital Survey on Patient Safety Culture (HSOPSC) was released by the Agency for Healthcare Research and Quality in November 2004. The survey is one of the most commonly applied instruments for the assessment of PSC. Moreover, it has been widely translated and validated in several languages and countries, including Belgium, England, Norway, Scotland, the Netherlands and Palestine. ${ }^{17-21} 2526$ The survey is intended to help hospitals assess the extent to which their cultures emphasise the importance of patient safety, encourage both the reporting and open discussion of errors and create an atmosphere of continuous learning and improvement.

The original HSOPSC consists of 42 items loading on 12 dimensions: 2 outcome dimensions and 10 safety dimensions. The 10 different PSC dimensions are expected to have strong relationships with the two composite outcome dimensions (frequency with events reported and overall perceptions of patient safety (OPS)) and the two single-item outcomes (patient safety grade and number of events reported (NER)). ${ }^{14}$ The four outcomes are self-reported patient safety outcomes.

Despite efforts to measure PSC in terms of dimensions and outcomes to improve patient safety, few studies have examined the specific predictive value of the dimensions in terms of HSOPSC outcomes. ${ }^{172027}$ Research to date has not detailed the relationships between the HSOPSC dimensions and its self-reported outcome measures across countries. Being part of this patient safety collaborative project between Palestinian and Belgian academic institutions allowed us to do this comparison. The two healthcare systems are different, one from developed countries (Belgium), having universal coverage, higher spending per capita, higher human resources ratios and system functioning in stable conditions compared with another system from a developing country (Palestine) that has a national insurance system with high out-ofpocket spending (40\%), especially for pharmaceuticals, lower physician density (1.6 in Palestine comparing with
2.96 in Belgium per 1000 inhabitants) and lower density of practising nurses (3.0-9.51 per 1000 population) ${ }^{28-30}$ Despite these differences, both countries have ongoing initiatives to improve patient safety of care. The governments of Belgium and Palestine promote the HSOPSC as a tool to support assessing PSC and providing baseline data to support the development of patient safety interventions or initiatives in hospitals. Our study included hospitals that are operated in both governmental and non-governmental sectors in Belgium and Palestine. We assumed that there were no staff-related differences that would influence the PSC between the two countries. Both governments have called for further investigations into the effectiveness of the HSOPSC within and between different cultural contexts. ${ }^{15}$ Thus, the first aim of this study was to determine which HSOPSC dimensions are associated with self-reported outcome measures. Second, we aimed to investigate the impact of cultural context on these associations. These findings offer further insights into the similarities and differences regarding associations between the PSC dimensions and outcomes of hospital workers in two very different cultural settings. The results also further attest to the value of the HSOPSC to increase patient safety in a range of settings.

\section{METHODS}

\section{Design, setting and sample}

A cross-sectional study design was used. In total, 90 acute Belgian hospitals and 13 Palestinian hospitals were included in the study. In Belgium, workshops were organised for participating hospitals in which the objectives and survey were explained. The Dutch and French validated versions of the HSOPSC were distributed organisation wide to 90 acute hospitals that participated in a federal patient safety programme between 2007 and 2009 (baseline PSC measurement ${ }^{152526}$ ). These hospitals comprised 58 Dutch-speaking hospitals, 31 Frenchspeaking hospitals and one that was both Dutch speaking and French speaking. In total, 68 hospitals used a paperbased survey, 15 used an electronic survey and 7 applied a mixed method for survey administration. Technical assistance was available during the periods of data collection. Hospitals were invited to participate in a Belgian comparative study on a voluntary, confidential and free-of-charge basis. A total of 91852 questionnaires were distributed, of which 47648 were returned (response rate 51.9\%). The comparative database was managed by a neutral academic institution and was not accessible to government authorities.

In Palestine, PSC baseline data were collected from September 2010 to August 2011 in 13 hospitals: 11 public hospitals and 2 general non-governmental hospitals situated in the West Bank. All hospitals were Arabic speaking and used a validated paper-based Arabic language survey. ${ }^{21}$ Workshops were organised at the participating hospitals to explain the objectives and survey. A paper version of the questionnaire was distributed, and participants were 
informed about the purpose of the study. They were also told that their participation was anonymous, voluntary and confidential. Technical assistance was accessible during the periods of data collection. A total of 3153 questionnaires were distributed, of which 1690 were returned (response rate $53.6 \%$ ), of which 1418 could be used for this study. The collected database was managed by an independent academic institution.

For both the Belgian and Palestinian samples, the survey was distributed anonymously to all healthcare staff that had direct (physicians, nurses, clinical staff, pharmacists, radiology staff and laboratory staff) or indirect (all hospital supervisors, managers, administrators and support and administrative staff) interaction with patients. The survey was self-administered and then placed in sealed envelopes without any respondent identification. Collection points/boxes were identified for returning the completed questionnaires.

For the purposes of this study, the Belgian sample was matched to the Palestinian sample by selecting a matched subsample based on staff position, years of experience at the hospital and number of hours worked per week. Specifically, we used the Palestinian set $(n=1418)$ to randomly (blind) select 1:1 matching participants regarding position, tenure and working hours from the Belgian sample. When more than one matching participant was identified, we randomly (blind) selected one of the participants that fitted the criteria. The matched sample was selected manually by the data management department.

The Belgian and Palestinian samples consisted of a total of 2836 healthcare staff (1418 respondents from each country). The subsample comprised the following staff categories: nurses $(38.6 \%)$, head nurses $(9.4 \%)$, nursing aid staff $(5.7 \%)$, physicians $(17.9 \%)$, pharmacists $(2.9 \%)$ and other healthcare staff (25.5\%). The sample hospitals ranged in size from small (fewer than 150 beds) to large (more than 200 beds). The research was fully supported and ethically approved by the Departments of Health of the Belgian and Palestinian governments, and all the hospitals that were approached participated in our study.

\section{Funding statement}

This research received no specific grant from any funding agency in the public, commercial or not-for-profit sectors.

\section{Instruments and measures}

The HSOPSC consists of 42 items and is designed to measure 10 PSC dimensions and 4 outcome measures. Items are scored on a 5-point Likert scale ranging from $1=$ 'strongly disagree' to $5=$ ='strongly agree' or from $1=$ 'never' to $5=$ 'always'. Respondents were also asked to provide some demographic information (eg, their work area/unit, staff position, whether they have direct interaction with patients). ${ }^{14}$ PSC dimensions and self-reported outcomes are shown in table 1.

Using the matched samples from both countries, the relationships between all dimensions and outcomes of the HSOPSC were assessed. Five-point response scales were used ( $1=$ 'strongly disagree' to $5=$ 'strongly agree').

\section{Independent variables}

For both samples, the PSC dimensions shown in table 1 were used. All PSC dimensions consist of multiple items.

\section{Dependent variables}

Four self-reported outcome measures were used as dependent variables (table 1). Two composite outcomes have multiple items scored on a 5-point Likert scale, of which the labels vary throughout the dimensions as follows: (1) frequency with events reported from $1=$ 'strongly disagree' to $5=$ ='strongly agree' and (2) overall perceptions of patient safety from $1=$ 'never' to $5=$ 'always'. Patient safety grade and number of events are ordinal, ranging from 1 to 5 ( $1=$ 'failing' to $5=$ 'excellent') and from 1 to $6(1=$ 'no event' to $6=$ 'more than 21 events'), respectively; both are single-item measures.

\section{Control variables}

The following controls were measured in both samples: staff position is categorical; years of working experience at the hospital is numerical, ranging from 1 to 6 (1='less than 1 year' to $6=$ '21 years or more'), and number of hours worked per week is numerical, ranging from 1 to 6 $(1=$ 'less than 20 hours per week to $6=$ ' 100 hours or more per week'). The control variables were selected based on previous research and/or their significant correlations with the outcomes. ${ }^{3132}$

\section{Statistical analysis}

A total of 2836 healthcare staff participated in the study. For the purposes of our analysis, negatively worded items were reversed coded so that a higher score reflected a more positive response. First, we conducted exploratory factor analyses (EFAs) using the Palestinian and matched Belgian data to test whether we could detect the same dimension structure in both samples. We aimed for acceptable levels of reliability (Cronbach's $\alpha \geq 0.60$ ) as recommended in the HSOPSC user guide. ${ }^{33}$ Based on the EFA, we optimised the model by defining 10 PSC dimensions instead of the original 12 HSOPSC dimensions in both the Palestinian and Belgian data. Four original dimensions were revealed as two dimensions. One combined 'communication openness' and 'feedback about error' in one dimension and the other combined 'teamwork across hospital units' and 'hospital handoffs and transitions' (table 2). The other two outcome measures were individual items: 'patient safety grade' and 'number of events reported'. Cronbach's $\alpha$ values for both the Palestinian and Belgian databases are presented in table 2.

Second, we conducted hierarchical regression analyses for both the Palestinian and Belgian samples using IBM SPSS V.20.0. Regression analyses are particularly suitable for analyses of variance in which we aim to test whether and to what extent the variance on the dependent variables (and as reported by the respondents) can be explained by the independent variables, which is reflected by $R^{2}$. By 
Table 1 Definitions of patient safety culture dimensions and outcomes

Items

\section{Patient safety culture dimensions}

Communication openness: staff freely speak up Staff will freely speak up if they see something that may negatively affect patient care.

if they see something that may negatively affect Staff feel free to question the decisions or actions of those with more authority.

a patient and feel free to question those with $\rightarrow$ Staff are afraid to ask questions when something does not seem right.

more authority.

Feedback and communication about errors: staff are informed about errors that happen, given feedback about changes implemented and discuss ways to prevent errors.

Hospital handoffs and transitions: important patient care information is transferred across hospital units and during shift changes.

Hospital management support for patient safety: hospital management provides a work climate that promotes patient safety and shows that patient safety is a top priority.

Non-punitive response to error: staff feel that their mistakes and event reports are not held against them and that mistakes are not kept in their file.

\section{Organisational learning-continuous} improvement: Mistakes have led to positive changes, and changes are evaluated for effectiveness.

Staffing: there is enough staff to handle the workload and work hours are appropriate to provide the best care for patients. promoting safety: supervisors/managers consider staff suggestions for improving patient safety, praise staff for following patient safety procedures and do not overlook patient safety problems.

Teamwork across hospital units: hospital units cooperate and coordinate with one another to provide the best care for patients.

Teamwork within hospital units: staff support each other, treat each other with respect and work together as a team.
Supervisor/manager expectations and actions

- We are given feedback about changes put into place based on event reports.

- We are informed about errors that happen in this unit.

- In this unit, we discuss ways to prevent errors from happening again.

- Things 'fall between the cracks' when transferring patients from one unit to another

- Important patient care information is often lost during shift changes.

- Problems often occur in the exchange of information across hospital units.

- Shift changes are problematic for patients in this hospital.

- Hospital management provides a work climate that promotes patient safety.

- The actions of hospital management show that patient safety is a top priority.

- Hospital management seems interested in patient safety only after an adverse event happens.

- Staff feel like their mistakes are held against them.

-When an event is reported, it feels like the person is being written up, not the problem.

- Staff worry that mistakes they make are kept in their file.

We are actively doing things to improve patient safety.

- Mistakes have led to positive changes here.

- After we make changes to improve patient safety, we evaluate their effectiveness.

- We have enough staff to handle the workload.

- Staff in this unit work longer hours than is best for patient care.

- We use more agency/temporary staff than is best for patient care.

- We work in 'crisis mode', trying to do too much, too quickly.

- My supervisor/manager offers praise when he/she sees a job done according to established patient safety procedures.

- My supervisor/manager seriously considers staff suggestions for improving patient safety.

Whenever pressure builds up, my supervisor/manager wants us to work faster, even if it means taking shortcuts.

- My supervisor/manager overlooks patient safety problems that happen repeatedly.

- There is good cooperation among hospital units that need to work together.

- Hospital units work well together to provide the best care for patients.

- Hospital units do not coordinate well with each other.

- It is often unpleasant to work with staff from other hospital units.

- People support one another in this unit.

-When a lot of work needs to be done quickly, we work together as a team to get the work done.In this unit, people treat each other with respect.

-When one area in this unit gets really busy, others help out.

\section{Self-reported outcome measures}

Frequency of events reported: mistakes of the following types are reported: (1) mistakes caught and corrected before affecting the patient ; (2) mistakes with no potential to harm the patient; and (3) mistakes that could harm the patient but do not.

Overall perceptions of patient safety: procedures and systems are good for the prevention of errors and there are minimal patient safety problems.

Patient safety grade: overall grade for patient safety for their work area/unit.

Number of events reported: the number of events reported over the past 12 months.
When a mistake is made but is caught and corrected before affecting the patient, how often is this reported?

When a mistake is made but has no potential to harm the patient, how often is this reported?

When a mistake is made that could harm the patient but does not, how often is this reported?

- Patient safety is never sacrificed to get more work done.

- Our procedures and systems are good for the prevention of errors.

- It is just by chance that more serious mistakes do not happen around here.

- We have patient safety problems in this unit.

- Please give your work area/unit in this hospital an overall grade for patient safety.

In the past 12 months, how many event reports have you filled out and submitted? 
Table 2 Percentage positive scores for patient safety dimensions and Cronbach's alpha of Hospital Survey on Patient Safety Culture (HSOPSC) in Palestine and Belgium

\begin{tabular}{|c|c|c|c|c|}
\hline & $\begin{array}{l}\text { Per cent positive } \\
\text { response Palestine }\end{array}$ & $\begin{array}{l}\text { Per cent positive } \\
\text { response } \\
\text { Belgium }\end{array}$ & $\begin{array}{l}\text { Cronbach's } \\
\text { alpha Palestine }\end{array}$ & $\begin{array}{l}\text { Cronbach's } \\
\text { alpha Belgium }\end{array}$ \\
\hline \multicolumn{5}{|l|}{ Patient safety culture dimensions } \\
\hline Teamwork within hospital units & 75 & 73 & 0.80 & 0.79 \\
\hline Organisational learning-continuous improvement & 64 & 49 & 0.73 & 0.61 \\
\hline $\begin{array}{l}\text { Supervisor/manager expectations and actions } \\
\text { promoting safety }\end{array}$ & 55 & 58 & 0.74 & 0.74 \\
\hline No punitive response to error & 17 & 38 & 0.63 & 0.69 \\
\hline Hospital management support for patient safety & 42 & 33 & 0.76 & 0.77 \\
\hline $\begin{array}{l}\text { Teamwork across hospital units and hospital } \\
\text { handoffs and transitions }\end{array}$ & 45 & 27 & 0.78 & 0.75 \\
\hline Staffing (staff) & 58 & 37 & 0.67 & 0.61 \\
\hline $\begin{array}{l}\text { Feedback and communication openness about } \\
\text { errors }\end{array}$ & 49 & 51 & 0.76 & 0.80 \\
\hline \multicolumn{5}{|l|}{ Patient safety culture outcomes } \\
\hline Frequency of event reporting & 39 & 44 & 0.87 & 0.86 \\
\hline Overall perceptions of safety & 55 & 47 & 0.75 & 0.73 \\
\hline Overall grade of patient safety & 49 & 39 & NA & NA \\
\hline Number of events reported & 45 & 69 & NA & NA \\
\hline
\end{tabular}

including hierarchical analyses, we are able to detect how much extra variance ( $R^{2}$ change) in the PSC outcomes is explained by introducing the PSC dimension in the analyses on top of the control variables. ${ }^{34}$ A composite average score was generated by calculating the average responses to the items within a dimension. As five-point response scales were used, the composite scores generated were any value between 1 and 5 . Regression analyses were conducted to determine the extent to which the PSC dimensions (modelled as independent variables) predicted the four self-reported dimensions (modelled as dependent variables). Multiple $\mathrm{R}\left(R^{2}\right)$ was used to indicate the proportion of variance explained by the model. An indication of the predictive value of the PSC dimensions was confirmed using standardised beta values $(\beta)$. A standardised beta coefficient is a standardised partial correlation coefficient that allows us to compare the strength of the effect of each predictor/independent variable in predicting the outcome/dependent variable, with higher absolute values of the beta coefficient indicating a stronger effect. Based on their significant correlation with the four outcomes over the Palestine and Belgian samples, the following controls were used in all regression analyses: experience at hospital (years), working hours (hours) and staff position. The control variables included in both countries were consistent to assure we could compare the results.

\section{Data sharing statement}

The data that support the findings of this study are available from the correspondent author on reasonable request and with permission of University of Leuven and University of Hasselt.

\section{Patient and public involvement}

Patient and public were not involved in the study.

\section{Ethical approval}

To ensure the privacy of the respondents, the survey was conducted anonymously. The researchers obtained ethical approval from the Departments of Health of the Belgian and Palestinian governments and institutional permits from the participating hospitals. Formal ethical approval and informed patient consent were not necessary for this type of study.

\section{RESULTS}

\section{Participants' characteristics}

Most participants were nurses $761(53.7 \%)$ and 254 (17.9\%) were physicians. Most of the participants had between 1 year and 5 years of experience in their current work unit. Details of the matched sample and participants' characteristics for both Palestinian and Belgian respondents are described in table 3 .

\section{Correlation between PSC dimensions and outcomes of the HSOPSC}

Table 4 presents the results of two correlation tables; namely, Palestinian correlations shown below the diagonal and Belgian correlations above. Preliminary 


\begin{tabular}{|c|c|c|c|}
\hline Characteristics & Whole sample & Palestinian sample $n=1418$ & Matched Belgian sample $n=1418$ \\
\hline \multicolumn{4}{|c|}{ Direct contact or interaction with patient } \\
\hline Yes, n (\%) & $2524(88.9)$ & $1284(90.5)$ & $1240(87.4)$ \\
\hline No, n (\%) & $312(11.0)$ & $134(9.4)$ & $178(12.5)$ \\
\hline \multicolumn{4}{|c|}{ Experience at current work area/unit } \\
\hline$<1$ year & $452(15.9)$ & $218(15.4)$ & $234(16.5)$ \\
\hline $1-5$ years & $1206(42.5)$ & $621(43.8)$ & $585(41.3)$ \\
\hline $6-10$ years & $552(19.5)$ & $250(17.6)$ & $302(21.3)$ \\
\hline $11-15$ years & $281(09.9)$ & $162(11.4)$ & $119(8.4)$ \\
\hline 16-20years & $184(06.5)$ & $81(5.7)$ & $104(7.3)$ \\
\hline$>21$ years & $160(05.6)$ & $86(6.1)$ & $74(5.2)$ \\
\hline \multicolumn{4}{|l|}{ Hospital size (beds) } \\
\hline Small (< 150), n (\%) & $700(24.7)$ & $612(43.1)$ & $88(6.2)$ \\
\hline Medium (150-249), n (\%) & $632(22.3)$ & $546(38.5)$ & $86(6.1)$ \\
\hline Large ( $\geq 250)$, n (\%) & $1504(53.0)$ & $260(18.4)$ & $1244(87.7)$ \\
\hline
\end{tabular}

Spearman's correlation analyses (table 4) revealed some differences between the Palestinian and Belgian samples. The analyses showed significant positive correlations between overall perceptions of patient safety (OPS) and most of the dimensions in both samples. Regarding the frequency of event reporting (FER), the data also showed a positive correlation with most of the dimensions in both samples. In both samples, most dimensions had a positive association with overall grade for patient safety (OGPS). Regarding the NER, the Palestinian sample had a significant and negative relationship with four dimensions: supervisor/manager expectations and actions promoting safety, non-punitive response to error, staffing and teamwork across hospital units and hospital handoffs and transitions. A negative correlation indicates the existence of an inverse relationship between two variables; that is, an increase in one variable is associated with a decrease in the other variable. This was also the case for the Belgian sample, with the NER found to be associated positively with organisational learning-continuous improvement, and negatively with support from hospital management for patient safety, staffing and teamwork across hospital units and during hospital handoffs and transitions.

\section{Hierarchical regression analyses}

Regression analyses were used to investigate the predictive value of the PSC dimensions regarding the four self-reported outcome measurements. The detailed results are shown in table 5, and the results are discussed generally below.

\section{Overall perceptions of safety}

The PSC dimensions explained $16 \%$ and $36 \%$ of the variance of OPS in the Palestinian and Belgian samples, respectively. OPS outcome was predicted in both countries by teamwork within hospital units, organisational learning-continuous improvement, supervisor/manager expectations and actions promoting safety, support from hospital management for patient safety, teamwork across hospital units and during hospital handoffs and transitions, and feedback and communication openness regarding errors. The standardised beta $(\beta)$ values ranged from 0.06 to 0.24 ( $\mathrm{p}$ values: $<0.05$ to $<0.001$ ). In addition to the previously mentioned predictors of OPS, staffing was a predictor for the Belgian sample only $(\beta=0.24, p<0.001)$.

\section{Frequency of event reporting}

In the Palestinian and Belgian samples, the PSC dimensions explained $22 \%$ and $18 \%$ of the variance of FER outcome, respectively. This outcome was predicted by teamwork across hospital units and during hospital handoffs and transitions, staffing and feedback and communication openness regarding errors with $\beta$ values ranging from 0.06 to 0.35 ( $p$ values $<0.05$ to $<0.001$ ). Organisational learning-continuous improvement $(\beta=0.14$, $\mathrm{p}<0.001)$ and hospital management support for patient safety $(\beta=0.10, \mathrm{p}<0.001)$ were also good predictors for the Palestinian sample.

\section{Overall grade on patient safety}

In total, $20 \%$ of the OGPS was predicted by PSC dimensions in the Palestinian sample and $33 \%$ in the Belgian sample. The results revealed two similar predictors in the two countries, namely supervisor/manager expectations and actions promoting safety and teamwork across hospital units and during hospital handoffs and transitions, with $\beta$-values ranging from 0.12 to $0.17(\mathrm{p}<0.001)$. In addition to the previously mentioned predictors, organisational learning-continuous improvement $(\beta=0.19, \mathrm{p}<0.001)$ and non-punitive response to error $(\beta=0.09, \mathrm{p}<0.001)$ were significant predictors of OGPS in Palestine. Furthermore, teamwork within hospital units $(\beta=0.11, \mathrm{p}<0.001)$, support from hospital management for patient safety 


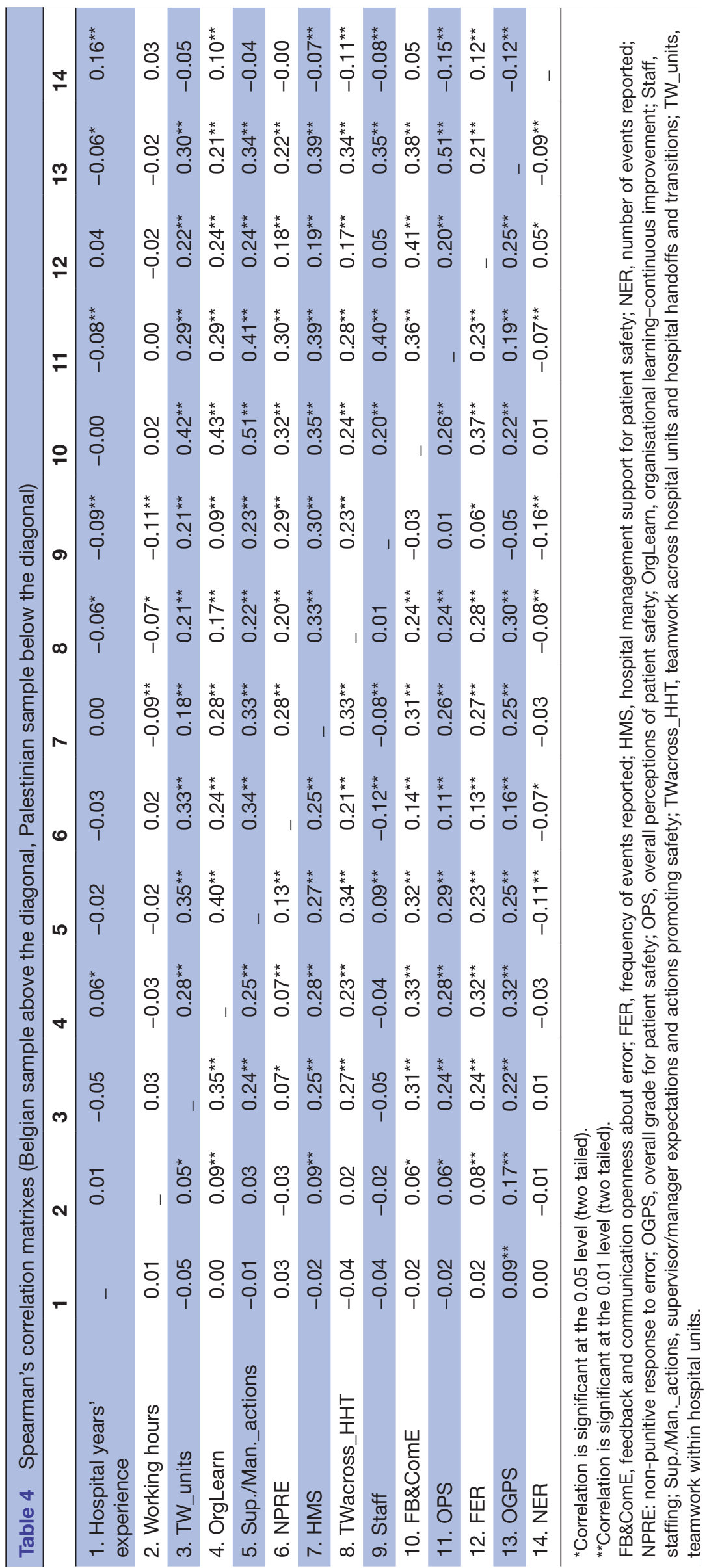




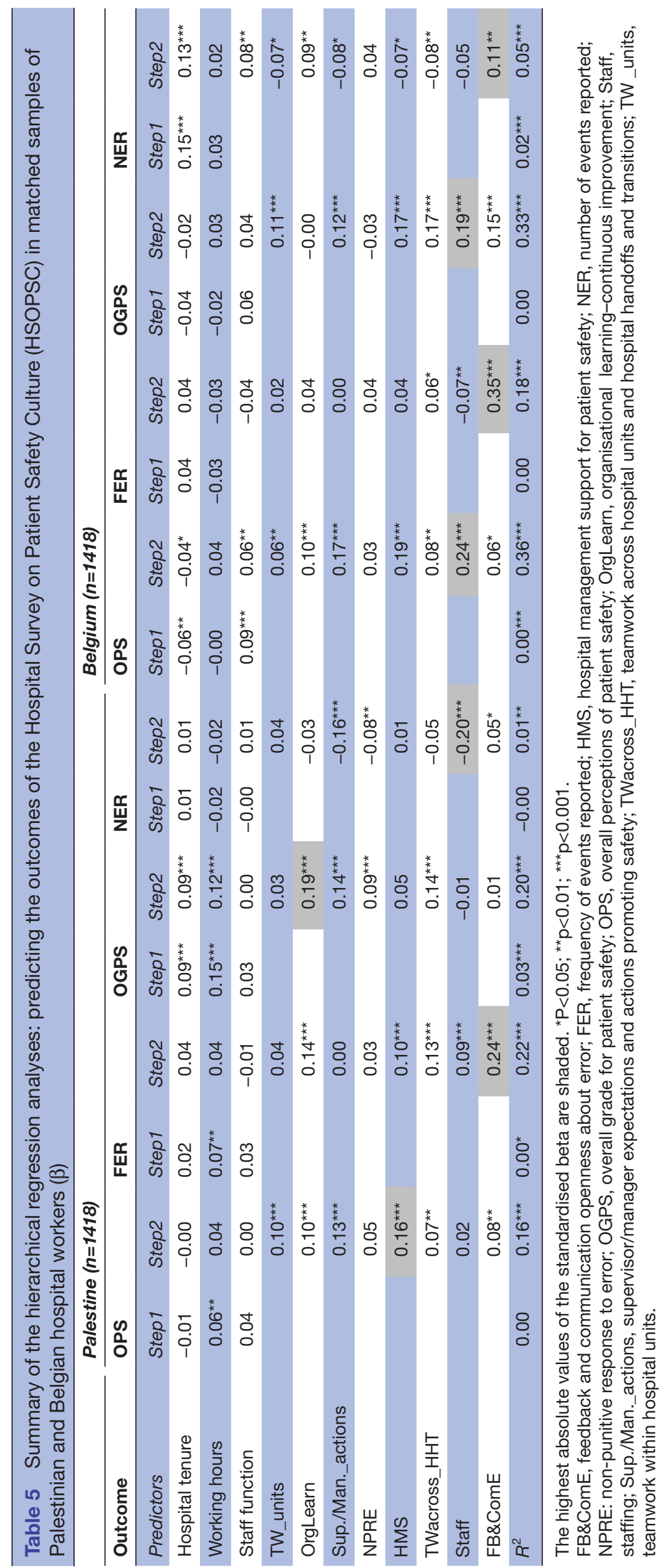


$(\beta=0.17, \mathrm{p}<0.001)$, staffing $(\beta=0.19, \mathrm{p}<0.001)$ and feedback and communication openness regarding errors $(\beta=0.15, p<0.001)$ were significant predictors of OGPS in Belgium.

\section{Number of events reported}

The PSC dimensions predicted only $1 \%$ of the NER in Palestine and 5\% in Belgium. Supervisor actions promoting safety and communication openness regarding errors were good predictors in both countries ( $\beta$ ranging from 0.05 to 0.16 , $p$ values ranging from $<0.05$ to $<0.001$ ). In Palestine, non-punitive response to error $(\beta=-0.08$, $\mathrm{p}<0.01)$ and staffing $(\beta=-0.20, \mathrm{p}<0.001)$ were also predictors of the NER. Moreover, teamwork within hospital units $(\beta=-0.07, p<0.05)$, organisational learning-continuous improvement $(\beta=0.09, \mathrm{p}<0.01)$, hospital management support for patient safety $(\beta=-0.07, \mathrm{p}<0.05)$, and teamwork across hospital units and during hospital handoffs and transitions $(\beta=-0.08,<0.01)$ were predictors in Belgium.

\section{DISCUSSION}

This study enabled us to draw conclusions regarding the extent to which each HSOPSC safety dimension contributes specifically to outcome dimensions. It is the first study to explore the predictive value of HSOPSC in matched samples from two different countries. As such, it provides information about: (A) the impact of the various PSC dimensions on patient safety outcomes and (B) cross-cultural differences in this respect between Palestinian and Belgian hospitals. Thus, our research provides an improved understanding of the influence of initiatives to improve specific outcome measures on PSC. Overall, our findings emphasise that the HSOPSC is a valid instrument that can be used to improve outcomes related to safe healthcare for patients. The results of our study demonstrate that at least two of the HSOPSC dimensions contribute to one of the self-reported outcome measures in each country. Thus, our findings attest to the value of the PSC dimensions regarding patient safety outcomes. Only one dimension, non-punitive response to error, was found to have no association with any of the outcome measures in Belgium. A possible explanation for this could be that this dimension has a low internal consistency level and, therefore, affected the assessment of its predictive value in the Belgian sample. Despite this finding, only a small number of differences were detected between the two samples.

The results obtained for both the Palestinian and Belgian samples showed that hospitals should focus on investing in interventions that enable feedback and enhance communication openness regarding errors, sustain teamwork within and across hospital units, maintain organisational learning-continuous improvement and improve hospital handoffs and transitions. These interventions will improve OPS. As such, our analyses and results explicitly reveal 'important' PSC dimensions in terms of safety outcomes, and these PSC dimensions are shown to be the same for both the Palestinian and Belgian hospitals. Under such circumstances, implementing strategies and tools such as TeamSTEPPS may improve teamwork within and across units, in addition to strengthening communication and feedback skills regarding errors to enable the establishment of a learning system based on previous mistakes. $^{33}$

Event reporting is fundamental to the detection of patient safety problems and represents a core prerequisite of effective clinical risk management. ${ }^{27}{ }^{35}$ This outcome is of particular importance because these items reflect the frequency of the actual reporting of an act, and the willingness to report unsafe events, with higher error reporting rates leading to a stronger culture of accountability. The results showed that, in both the Palestinian and Belgian samples, this outcome is influenced mainly by maintaining open lines of information and communication in the unit. These observations are consistent with those reported by Pfeiffer and Manser. ${ }^{27}$ Moreover, improvements in staffing and teamwork may also influence this outcome. Palestinian respondents found that managers who consider patient safety to be a top priority and build constructive learning systems based on previous mistakes encourage their staff to report adverse events. This result is also commonly reported in the published literature. $^{135}$

The OGPS was found to be particularly significant in building a constructive learning system based on previous mistakes in Palestine and in improving staffing levels in Belgium. In other words, although our results revealed many similarities between the matched set of Palestinian and Belgian healthcare professionals, we also found cultural differences regarding OGPS. In accordance with previous studies, having enough staff ${ }^{36}$ and opportunities to learn from previous mistakes ${ }^{6}$ increased the likelihood of staff reporting good or excellent safety grades in Belgium. Other contributing PSC dimensions are maintaining manager expectations and actions in promoting safety, improving teamwork across hospital units and supporting hospital handoffs and transitions in both countries. Our study also revealed that, in Palestine, a higher score for OGPS relates to greater support regarding non-punitive response to error. Additionally, initiatives to improve teamwork within units, support from hospital management for patient safety, staffing and feedback and communication openness regarding errors may also act to improve OGPS in Belgium.

As PSC dimensions explained only $1 \%$ (Palestine) and $5 \%$ (Belgium) of the NER, influencing PSC dimensions will have less impact on patient safety outcomes compared with other PSC outcomes $\left(R_{\text {OPS-Palestine }}^{2}=16 \% ; R_{\text {OPS-Bel- }}^{2}\right.$ gium $=36 \% ; R_{\text {FER-Palestine }}^{2}=22 \% ; \quad R_{\text {FER-Belgium }}^{2}=18 \% ; R_{\text {OGPS-Pales- }}^{2}$ tine $\left.=20 \% ; R_{\text {OGPS-Belgium }}^{2}=33 \%\right)$. As such, the HSOPSC seems to be particularly effective for the prediction of OPS, FER and OGPS, in both the Belgian and Palestinian samples. It can be speculated that the finding that NER is less well explained through the PSC dimensions is caused by the 
broad scope of the HSOPSC PSC dimensions regarding patient safety, which consequently renders this instrument less effective for this very specific outcome. A further explanation could be that our results stem not from a low predictive value but from the under-reporting of adverse events, which would result in a low mean and low variance regarding this outcome dimension. A final explanation could be that respondents found it difficult to answer this question based on experience, as they may not themselves know how many events have been reported. Therefore, hospitals seeking to invest in patient safety must measure NER and should link the PSC dimensions to objective measurements of NER.

To summarise, our results suggest that improved self-reported outcome measures regarding patient safety in both Palestine and Belgium are more likely to be achieved through better teamwork across units and during hospital handoffs and transitions, encouraging feedback and communications regarding errors and implementing actions to promote safety. Furthermore, improving teamwork within hospital units, providing a work climate that promotes the adoption of safety as a top priority by hospital management and resolving staffing problems will also influence most outcome measures in Belgium. ${ }^{37}{ }^{38}$ In Palestine, bringing errors to the attention of managers and other staff and using mistakes as valuable learning opportunities ${ }^{3}$ may also have an impact on safety outcome measures.

\section{Limitations and future research}

The current study has some methodological limitations that should be noted. First, the study used a cross-sectional design; therefore, claims of causal relationships are not possible. Second, as the present study relied exclusively on subjective measures that reflect the willingness of respondents to report events, and more specifically, the reporting of near misses, the results may be distorted by the common method bias of these self-reported outcome measures. Future research should examine the relationship between PSC dimensions and actual adverse event rates to clarify this relationship by linking objective data with PSC dimensions. Nevertheless, this study is the first to provide further insights into the value of the HSOPSC in terms of: (A) the impact of the PSC dimensions on patient safety outcomes and (B) in different cultural settings. Third, the associations between the safety and PSC outcomes of the HSOPSC were investigated using a matched sample (to rule out possible bias due to sample differences between the two countries) using linear regression analyses. However, we cannot ignore the limitation of this design, which uses the most influential variables that are, in fact, approximations made from the researchers' perspective. These assumptions might be incorrect and could lead to the introduction of major confounding variables. Despite these methodological restrictions, previous research has shown that matched samples designs are useful, allowing researchers to conduct streamlined and focused research while maintaining a good degree of validity. ${ }^{39}$ Another possible concern is that most respondents were nurses and other health professionals; thus, the results may reflect the personal perceptions of the respondents and affect our association results. However, we should not forget that nurses are the most highly represented staffing group in hospitals and that our sample also included physicians, pharmacists, administrative and quality and safety staff. Finally, the Cronbach's $\alpha$ values for some of the composite scores measuring PSC were low $(\alpha<0.70)$, which may affect the correlation results, as in the Palestinian sample where all PSC dimensions were positively associated with the OGPS (except staffing). This specific dimension has shown a low internal consistency in most psychometric evaluation studies of the HSOPSC, ${ }^{21} 33$ indicating that it is necessary to review and update the items of this dimension to improve its internal consistency. Our results further attest to the international and cross-cultural validity of the HSOPSC. The relationships between the PSC and self-reported outcomes in other countries will provide further evidence in this regard.

\section{Conclusion}

We found that perceptions of staffing and feedback and communication regarding errors were important predictive dimensions of PSC self-reported outcome measures in both countries. However, we also found some contradictory results in our matched sample. Future research should focus on enriching the evidence of the linking of safety culture and hard patient safety outcomes in order to assess the practical validity of safety culture surveys. The divergences of patient safety perceptions in both countries implicate the need of local priority setting and a tailor-made approach for improvement strategies in hospitals. A great challenge lies in the field of implementation science, testing the effectiveness of safety culture strategies.

\section{Author affiliations}

${ }^{1}$ Population Health Department, Hospital - MNG-HA, King Abdullah International Medical Research Center, King Saud Bin Abdulaziz University for Health Sciences, Riyadh, Saudi Arabia

${ }^{2}$ Leuven Institute for Healthcare Policy, School of Public Health \& Primary Care, KU Leuven, Leuven, Belgium

${ }^{3}$ Department of Work and Organisation Studies, KU Leuven, Brussels, Belgium ${ }^{4}$ Leuven Institute for Healthcare Policy, School of Public Health \& Primary Care, Department of Quality Management, UZ Leuven, KU Leuven, Leuven, Belgium ${ }^{5}$ Department of Health Policy \& Management, Faculty of Public Health, Al-Quds University, Jerusalem, Palestine

${ }^{6}$ OrganizationalPsychology, research group Work, Organizational and

PersonnelPsychology, KU Leuven, Leuven, Belgium

${ }^{7}$ Faculty of Medicine and Life Sciences, Hasselt University, Hasselt, Belgium

Acknowledgements We particularly acknowledge all participating hospitals and their staff who devoted time to completing the surveys. We extend our gratitude to the Palestinian and Belgian governments for their cooperation and supporting this research.

Contributors SN performed the overall statistical analyses and drafted the manuscript. EB actively contributed to the statistical analyses, the interpretation of the results and to the preparation of the manuscript. MH coordinated data collection (Palestine) and critically revised the manuscript. ArV and ME participated in critically revising the manuscript for important intellectual content. WalS and 
WarS participated in the analyses and interpretation of the data and contributed to the preparation of the manuscript. $\mathrm{KV}$ and $\mathrm{JH}$ were involved in the study design and revised the manuscript critically. JH coordinated the data collection (Belgium). AnV was involved in the study design, manuscript outline and contributed to the manuscript and data preparation (Belgian data). All authors have read and approved the final manuscript.

Funding The authors have not declared a specific grant for this research from any funding agency in the public, commercial or not-for-profit sectors.

Competing interests None declared.

Patient consent Not required.

Provenance and peer review Not commissioned; externally peer reviewed.

Data sharing statement The data that support the findings of this study are available from the correspondent author on reasonable request and with permission of University of Leuven and University of Hasselt.

Open access This is an open access article distributed in accordance with the Creative Commons Attribution Non Commercial (CC BY-NC 4.0) license, which permits others to distribute, remix, adapt, build upon this work non-commercially, and license their derivative works on different terms, provided the original work is properly cited, appropriate credit is given, any changes made indicated, and the use is non-commercial. See: http://creativecommons.org/licenses/by-nc/4.0/.

\section{REFERENCES}

1. Schwendimann R, Zimmermann N, Küng K, et al. Variation in safety culture dimensions within and between US and Swiss Hospital Units: an exploratory study. BMJ Qual Saf 2013;22:32-41.

2. Donaldson LJ, Fletcher MG. The WHO World Alliance for Patient Safety: towards the years of living less dangerously. Med $J$ Aust 2006;184:S69e72.

3. Najjar S, Hamdan M, Euwema MC, et al. The Global Trigger Tool shows that one out of seven patients suffers harm in Palestinian hospitals: challenges for launching a strategic safety plan. Int J Qual Health Care 2013;25:640-7 https://doi.org/.

4. Institute of Medicine. To err is human: Building a safer health system. Washington, D.C: The National Academy press, 2000.

5. Wakefield JG, Jorm CM. Patient safety -- a balanced measurement framework. Aust Health Rev 2009;33:382-9.

6. Stone PW, Gershon RR. Nurse work environments and occupational safety in intensive care units. J Nurs Adm 2009;39:S27-S34.

7. Mardon RE, Khanna K, Sorra J, et al. Exploring relationships between hospital patient safety culture and adverse events. J Patient Saf 2010;6:226-32.

8. Singer S, Lin S, Falwell A, et al. Relationship of safety climate and safety performance in hospitals. Health Serv Res 2009;44:399-421.

9. Kotter JP, Heskett JL. Corporate Culture and Performance. New York: Free Press, 1992.

10. Carman JM, Shortell SM, Foster RW, et al. Keys for successful implementation of total quality management in hospitals. Health Care Manage Rev 2010;35:283-93.

11. Institute of Medicine. Crossing the Quality Chasm: A New Health System for the 21st Century. Washington, DC: National Academy Press, 2001.

12. Edmondson A. Psychological Safety and Learning Behavior in Work Teams. Adm Sci Q 1999;44:350-83.

13. Hansen LO, Williams MV, Singer SJ. Perceptions of hospital safety climate and incidence of readmission. Health Serv Res 2011;46:596-616.

14. Sorra J, Famolaro T, Dyer N, et al. Hospital Survey on Patient Safety Culture: 2012 User Comparative Database Report. Prepared by Westat, Rockville, MD, under Contract No. HHSA $290200710024 \mathrm{C}$ Rockville, MD: Agency for Healthcare Research and Quality, 2012.

15. Vlayen A, Hellings $\mathrm{J}$, Claes $\mathrm{N}$, et al. A nationwide hospital survey on patient safety culture in Belgian hospitals: setting priorities at the launch of a 5-year patient safety plan. BMJ Qual Saf 2012;21:760-7.

16. Deilkås $E$, Hofoss D. Patient safety culture lives in departments and wards: multilevel partitioning of variance in patient safety culture. BMC Health Serv Res 2010;10:85.
17. Sorra JS, Dyer N. Multilevel psychometric properties of the AHRQ hospital survey on patient safety culture. BMC Health Serv Res 2010;10:199.

18. Waterson P, Griffiths P, Stride C, et al. Psychometric properties of the Hospital Survey on Patient Safety Culture: findings from the UK. Qual Saf Health Care 2010;19:e2.

19. Sarac C, Flin R, Mearns K, et al. Hospital survey on patient safety culture: psychometric analysis on a Scottish sample. BMJ Qual Saf 2011;20:842-8.

20. Olsen E. Reliability and validity of the Hospital Survey on Patient Safety Culture at a Norwegian hospital. In: Øvretveit J, Sousa P, eds. Quality and Safety Improvement Research: Methods and Research Practice from the International Quality Improvement Research Network (QIRN). Lisbon: National School of Public Health, 2008:173-86.

21. Najjar S, Hamdan M, Baillien E, et al. The Arabic version of the hospital survey on patient safety culture: a psychometric evaluation in a Palestinian sample. BMC Health Serv Res 2013;13:193.

22. Colla JB, Bracken AC, Kinney LM, et al. Measuring patient safety climate: a review of surveys. Qual Saf Health Care 2005;14:364-6.

23. Sexton JB, Helmreich RL, Neilands TB, et al. The Safety Attitudes Questionnaire: psychometric properties, benchmarking data, and emerging research. BMC Health Serv Res 2006;6:44.

24. Sammer CE, Lykens K, Singh KP, et al. What is patient safety culture? A review of the literature. J Nurs Scholarsh 2010;42:156-65.

25. Hellings J, Schrooten W, Klazinga $\mathrm{N}$, et al. Challenging patient safety culture: survey results. Int J Health Care Qual Assur 2007;20:620-32.

26. Vlayen A, Hellings J, Claes N, et al. Measuring safety culture in belgian psychiatric hospitals: validation of the dutch and French translations of the hospital survey on patient safety culture. $J$ Psychiatr Pract 2015;21:124-39.

27. Pfeiffer $\mathrm{Y}$, Manser T. Development of the German version of the Hospital Survey on Patient Safety Culture: Dimensionality and psychometric properties. Saf Sci 2010;48:1452-62.

28. West Bank and Gaza Public Expenditure Review of the Palestinian Authority. Towards Enhanced Public Finance Management and Improved Fiscal Sustainability. Washington DC: World Bank, 2016. http://documents.worldbank.org/curated/en/ 320891473688227759/pdf/ACS18454-REVISED-FINAL-PERSEPTEMBER-2016-FOR-PUBLIC-DISCLOSURE-PDF.pdf. (accessed 18, May 2018)

29. WHO. Country Cooperation Strategy WHO - BELGIUM 2016-2022. http://www.euro.who.int/ data/assets/pdf file/0009/329778/CCS Belgium-2016-2022-en.pdf (Accessed 18, May 2018).

30. Palestinian Central Bureau of Statistics. Statistics from Palestine. https://thisweekinpalestine.com/wp-content/uploads/2014/11/ Statistics-from-Palestine.pdf (Accessed May 18, 2018).

31. El-Jardali F, Jaafar M, Dimassi H, et al. The current state of patient safety culture in Lebanese hospitals: a study at baseline. Int J Qual Health Care 2010;22:386-95.

32. Hickner J, Smith SA, Yount N, et al. Differing perceptions of safety culture across job roles in the ambulatory setting: analysis of the AHRQ Medical Office Survey on Patient Safety Culture. BMJ Qual Saf 2016;25:588-94.

33. Sorra JS, Nieva VF. Hospital Survey on Patient Safety Culture. AHRQ Publication No. 04-0041. Rockville, MD: Agency for Healthcare Research and Quality 2004.

34. Hayes AF. Introduction to mediation, moderation, and conditional process analysis: A regression-based approach. New York, NY, US: Guilford Press, 2017.

35. Westrum R. A typology of organisational cultures. Qual Saf Health Care 2004;13 Suppl 2(Suppl. 2):ii22-ii27.

36. Aiken LH, Sermeus W, Van den Heede K, et al. Patient safety, satisfaction, and quality of hospital care: cross sectional surveys of nurses and patients in 12 countries in Europe and the United States. BMJ 2012;344:e1717.

37. Keller SM. Effects of extended work shifts and shift work on patient safety, productivity, and employee health. Aaohn J 2009;57:497-502.

38. Mark BA, Harless DW, Berman WF. Nurse staffing and adverse events in hospitalized children. Policy Polit Nurs Pract 2007;8:83-92.

39. Stuart EA. Matching methods for causal inference: A review and a look forward. Stat Sci 2010;25:1-21. 